\title{
NONLINEAR STABILITY OF RAREFACTION WAVES FOR A VISCOELASTIC MATERIAL WITH MEMORY
}

\author{
HARUMI HATTORI
}

\begin{abstract}
In this paper we will discuss the stability of rarefaction waves for a viscoelastic material with memory. The rarefaction waves for which the stability is tested are not themselves solutions to the integrodifferential equations (1.1) governing the viscoelastic material. They are solutions to a related equilibrium system of conservation laws given by (1.11). We shall show that if the forcing term and the past history are small and if the initial data are close to the rarefaction waves, the solutions to (1.1) will approach the rarefaction waves in sup norm as the time goes to infinity.
\end{abstract}

\section{INTRODUCTION}

In this paper, we will discuss the nonlinear stability of rarefaction waves for a system,

$$
V_{t}=U_{x}, \quad U_{t}=\sigma(V)_{x}+\int_{-\infty}^{t} a^{\prime}(t-\tau) \phi(V)_{x} d \tau+f(x, t),
$$

with the initial data

$$
\begin{gathered}
(U(x, 0), V(x, 0))=\left(U_{0}(x), V_{0}(x)\right), \quad x \in R, \\
\lim _{x \rightarrow \pm \infty}\left(U_{0}(x), V_{0}(x)\right)=\left(U_{ \pm}, V_{ \pm}\right) .
\end{gathered}
$$

The above system can be derived from

$$
w_{t t}=\sigma\left(w_{x}\right)_{x}+\int_{-\infty}^{t} a^{\prime}(t-\tau) \phi\left(w_{x}(x, \tau)\right)_{x} d \tau+f(x, t),
$$

by setting $U=w_{t}$ and $V=w_{x}$. In this paper, for $\sigma$ and $\phi$ we require that

$$
\begin{array}{lll}
\sigma \in C^{3}(R), & \sigma^{\prime}>0, & \sigma^{\prime \prime}>0, \\
\phi \in C^{3}(R), & \phi^{\prime}>0, & \phi^{\prime \prime}>0 .
\end{array}
$$

For $a(t)$ we assume

$$
a, a^{\prime}, a^{\prime \prime} \in L^{1}(0, \infty), \quad a \text { is strongly positive definite on }(0, \infty) .
$$

Furthermore, we assume

$$
\int_{0}^{\infty} t|a(t)| d t<\infty, \quad\left(a^{\prime}\right)^{\wedge}(z) \neq 0 \quad \forall z \in \Pi,
$$

Received by the editors June 19, 1990 and, in revised form, April 10, 1992.

1980 Mathematics Subject Classification (1985 Revision). Primary 35L60, 70A10; Secondary $35 \mathrm{~L} 65$.

Key words and phrases. Hyperbolic system, rarefaction wave, viscoelasticity. 
where $\wedge$ means the Laplace transform and $\Pi=\{z \in C \mid \operatorname{Re} z \geq 0\}$. The assumption (1.5) implies

$$
a(0)>0, \quad a^{\prime}(0)<0 .
$$

In what follows, we assume without loss of generality

$$
a^{\prime}(0)=-1 \text {. }
$$

Integrating the integral term in (1.1) we obtain

$$
\left\{\begin{array}{l}
V_{t}=U_{x}, \\
U_{t}=\chi(V)_{x}+\int_{0}^{t} a(t-\tau) \phi(V)_{x t} d \tau+a(t) \phi\left(V_{0}\right)_{x}+h(x, t),
\end{array}\right.
$$

where

$$
\chi(V)=\sigma(V)-a(0) \phi(V)
$$

and

$$
h(x, t)=\int_{-\infty}^{0} a^{\prime}(t-\tau) \phi(V)_{x} d \tau+f(x, t) .
$$

In order that the equilibrium system

$$
V_{t}=U_{x}, \quad U_{t}=\chi(V)_{x},
$$

be hyperbolic and genuinely nonlinear, we assume that

$$
\chi^{\prime}>0, \quad \chi^{\prime \prime}>0 \text {. }
$$

The condition $\chi^{\prime}>0$ means in particular that the equilibrium stress modulus is positive.

As the form of (1.9) suggests, the asymptotic behavior of the solution for (1.9) and (1.2) is closely related to the Riemann problem for (1.11) with

$$
(U, V)(x, 0)=\left(U_{0}^{r}, V_{0}^{r}\right)(x)= \begin{cases}\left(U_{-}, V_{-}\right), & x<0, \\ \left(U_{+}, V_{+}\right), & x>0 .\end{cases}
$$

We should note that system (1.1) is not invariant under dilations of coordinates while system (1.11) is.

For each constant state, we define the rarefaction curves $R_{1}$ and $R_{2}$ through $\left(U_{-}, V_{-}\right)$in a neighborhood $\omega$ of $\left(U_{-}, V_{-}\right)$by

$$
\begin{aligned}
& R_{1}\left(U_{-}, V_{-}\right)=\left\{(U, V) \in \omega \mid U=U_{-}-\int_{V_{-}}^{V} \lambda_{1}(s) d s, \text { with } U \leq U_{-}\right\} \\
& R_{2}\left(U_{-}, V_{-}\right)=\left\{(U, V) \in \omega \mid U=U_{-}-\int_{V_{-}}^{V} \lambda_{2}(s) d s, \text { with } U \leq U_{-}\right\}
\end{aligned}
$$

where $\lambda_{1}(s)=-\sqrt{\chi^{\prime}(s)}$ and $\lambda_{2}(s)=\sqrt{\chi^{\prime}(s)}$. In (1.14) $R_{1}$ (resp. $\left.R_{2}\right)$ is the state which can be connected through $\left(U_{-}, V_{-}\right)$by the backward (resp. forward) rarefaction wave. We also define $R R$ as a part of $\omega$ such that $(1.15)$

$$
R R\left(U_{-}, V_{-}\right)=\left\{(U, V) \in \omega \mid U \leq U_{-}-\int_{V_{-}}^{V} \lambda_{1}(s) d s, U \leq U_{-}-\int_{V_{-}}^{V} \lambda_{2}(s) d s\right\} .
$$


Define $\delta=\left|U_{+}-U_{-}\right|+\left|V_{+}-V_{-}\right|$. Then, there exists a positive constant $\delta_{0}$ such that if $\left(U_{+}, V_{+}\right) \in R R\left(U_{-}, V_{-}\right)$and $\delta \leq \delta_{0}$, then there is a unique $\left(U_{m}, V_{m}\right) \in R_{1}\left(U_{-}, V_{-}\right)$satisfying $\left(U_{+}, V_{+}\right) \in R_{2}\left(U_{m}, V_{m}\right)$. In what follows, we discuss the case when $\left(U_{+}, V_{+}\right) \in R R\left(U_{-}, V_{-}\right)$and $\delta \leq \delta_{0}$. Then, the solution of the Riemann problem (1.11), (1.13) consists of centered rarefaction waves. This solution will be denoted by $\left(U^{r}, V^{r}\right)(x / t)$. Our goal is to show that the solution of (1.1) and (1.2) approaches the rarefaction wave $\left(U^{r}, V^{r}\right)(x / t)$ as $t \rightarrow+\infty$ provided that the initial data are suitably close to $\left(U_{0}^{r}, V_{0}^{r}\right)$. More precisely, we assume

$$
U_{0}-U_{0}^{r}, V_{0}-V_{0}^{r}, U_{0 x}, V_{0 x}, U_{0 x x}, V_{0 x x} \in L^{2}(R)
$$

and set

$$
\begin{aligned}
\Sigma_{0}= & \int_{-\infty}^{\infty}\left\{\left(U_{0}-U_{0}^{r}\right)^{2}+\left(V_{0}-V_{0}^{r}\right)^{2}+U_{0 x}^{2}+V_{0 x}^{2}+U_{0 x x}^{2}+V_{0 x x}^{2}\right\} d x \\
& +\left|U_{+}-U_{-}\right|+\left|V_{+}-V_{-}\right| .
\end{aligned}
$$

For $h(x, t)$ we assume

$$
\begin{aligned}
& h, h_{x}, h_{t} \in C\left([0, \infty) ; L^{2}(R)\right) \cap L^{\infty}\left([0, \infty) ; L^{2}(R)\right), \\
& \|h\|_{L^{1}}^{4 / 3} \in L^{1}[0, \infty), h, h_{x}, h_{t}, h_{x t} \in L^{2}\left([0, \infty) ; L^{2}(R)\right)
\end{aligned}
$$

and set

$$
\begin{aligned}
H= & \sup _{t \geq 0} \int_{-\infty}^{\infty}\left\{h^{2}+h_{x}^{2}+h_{t}^{2}\right\}(x, t) d x \\
& +\int_{0}^{\infty} \int_{-\infty}^{\infty}\left\{h^{2}+h_{x}^{2}+h_{t}^{2}+h_{x t}^{2}\right\}(x, t) d x d t \\
& +\int_{0}^{\infty}\left(\int_{-\infty}^{\infty}|h(x, t)| d x\right)^{4 / 3} d t
\end{aligned}
$$

Then, we have the following theorem.

Theorem 1.1. For each fixed $\left(U_{-}, V_{-}\right)$, there exists a positive constant $\varepsilon_{0}$ such that if $\left(U_{+}, V_{+}\right) \in R R\left(U_{-}, V_{-}\right)$and $\delta+\Sigma_{0}+H<\varepsilon_{0}$, then the initial value problem (1.1), (1.2) has a unique global solution in time satisfying

$$
\begin{array}{r}
U-U^{r}, V-V^{r}, U_{t}, V_{t}, V_{x}, U_{t t}, V_{t t}, V_{x t}, V_{x x} \\
\in C\left([0, \infty) ; L^{2}(R)\right) \cap L^{\infty}\left([0, \infty) ; L^{2}(R)\right) .
\end{array}
$$

Furthermore, the solution to (1.1), (1.2) will approach the equilibrium rarefaction waves as $t$ goes to infinity in the maximum norm, namely

$$
\sup _{x \in R}\left|(U, V)(x, t)-\left(U^{r}, V^{r}\right)(x / t)\right| \rightarrow 0 \text { as } t \rightarrow \infty \text {. }
$$

Remark 1.1. It should be noted that system (1.1) is not invariant under dialations of coordinates, and thus the rarefaction waves for which the stability is tested are not solutions to system (1.1).

Remark 1.2. The condition (1.17) implies that the rarefaction waves, the initial perturbations, and the past history should be weak.

To prove this theorem we first approximate $\left(U^{r}, V^{r}\right)$ by a smooth function $(\varphi, \psi)$. This will be described in $\S 2$. In this section, we also introduce the new 
variables $u=U-\varphi, v=V-\psi$, which are perturbations from $(\varphi, \psi)$. In $\S 3$, we discuss the properties of the kernels associated with the kernel $a(t)$. In $\S 4$ we will state Proposition 4.1 for $(u, v)$, which will imply Theorem 1.1. This will be shown in Proposition 4.2. The proof of Proposition 4.1 will be carried out in $\S 5$. The proof is based on the energy method and along the line of Hrusa and Nohel [4].

System (1.1) describes a one-dimensional motion of an unbounded, homogeneous, viscoelastic bar. The integral term represents the memory effect and induces subtle dissipation. The global existence of a classical solution for small initial data was obtained by various authors. In the case when $\phi=\sigma$ MacCamy [9] discussed the initial-boundary value problem and Dafermos and Nohel [1] discussed both the initial and the initial-boundary value problems. In the case when $\phi \neq \sigma$ Dafermos and Nohel [2] discussed the initial boundary value problem and Hrusa and Nohel [4] discussed the pure initial value problem. In the initial value problem, they prove: Under suitable assumptions on the material functions, the kernel, and the forcing term and for sufficiently small data belonging to $H^{2}$, the Cauchy problem $(1.1),(1.2)$ has a unique solution $(U, V)$ for $0 \leq t \leq \infty$; moreover, $U, V, U_{x}, U_{t}, V_{x}, V_{t} \rightarrow 0$ as $t \rightarrow \infty$ uniformly in $x$. As they assume that the initial data are in $H^{2}$, the initial data $U_{0}$ and $V_{0}$ approach zero as $x \rightarrow \pm \infty$. The case when $U_{0}$ and $V_{0}$ approach nonzero constant states as $x \rightarrow \pm \infty$ has not been discussed yet. In this paper we discuss such a case when the initial data are perturbations from rarefaction waves.

As for the Riemann problem, the Riemann data problem for the scalar equations modeling (1.1) has been discussed by MacCamy [10] and Greenberg and Hsiao [3].

The stability of rarefaction waves has been studied for viscous hyperbolic conservation laws $[7,11,13,14]$ and hyperbolic conservation laws with relaxation by the energy method [5]. Therefore, it is of interest to extend the result to the case where the dissipation is given by the fading memory. We should note that as the rarefaction waves for which the stability is studied, we choose the equilibrium rarefaction waves for the system constructed from the equilibrium characteristics, $\lambda= \pm \sqrt{\chi^{\prime}}$, and not the instantaneous characteristics $\lambda= \pm \sqrt{\sigma^{\prime}}$. The significance of the equilibrium characteristics is discussed in [6] in conjunction with travelling waves. It is also interesting to understand the importance of equilibrium characteristics for the rarefaction waves. In this paper we discuss the case when $\sigma \neq \phi$.

\section{SMOOTH APPROXIMATE SOLUTION OF THE RIEMANN PROBLEM}

In this section, using the idea of Matsumura and Nishihara [11], we will construct a smooth approximate solution to $\left(U^{r}, V^{r}\right)$. In what follows, we will use $\|\cdot\|$ to denote the $L^{2}$ norm and $\|\cdot\|_{i}$ to denote the $H^{i}$ norm unless it is explicitly specified.

Consider the Riemann problem for the simplest equation

$$
\begin{gathered}
w_{t}+w w_{x}=0, \\
w(x, 0)= \begin{cases}w_{-}, & x<0, \\
w_{+}, & x>0\end{cases}
\end{gathered}
$$

We assume that $w_{-}<w_{+}$. Then, the solution to $(2.1)$ is given by the centered 
rarefaction wave

$$
w^{r}(\xi)= \begin{cases}w_{-}, & \xi \leq w_{-}, \\ \xi, & w_{-} \leq \xi \leq w_{+}, \\ w_{+}, & \xi \geq w_{+},\end{cases}
$$

where $\xi=x / t$. We approximate this solution by the solution of the following problem

$$
w_{t}+w w_{x}=0, \quad w(x, 0)=w_{0}(x),
$$

where $w_{0}$ is given by

$$
w_{0}(x)=\frac{w_{+}+w_{-}}{2}+\frac{\left(w_{+}-w_{-}\right)}{2} \tanh x
$$

The choice of $w_{0}(x)$ is rather arbitrary. However, it makes the following estimates easier.

Lemma 2.1. The problem (2.4) has a unique smooth global solution in time satisfying the following

(i) $w_{-} \leq w(x, t) \leq w_{+}, w_{x}>0$ for all $x \in R$ and $t \geq 0$.

(ii) For $1 \leq p \leq+\infty$, there exists $a C_{p}$ such that for all $|\tilde{w}| \geq 0$ and $t \geq 0$

$$
\left\|w_{x}\right\|_{L^{p}} \leq C_{p} \min \left(|\tilde{w}|,|\tilde{w}|^{1 / p} t^{-1+1 / p}\right)
$$

where $|\tilde{w}|=w_{+}-w_{-}$.

(iii) For $1 \leq p \leq+\infty$, there exists a $C_{p}$ such that for all $|\tilde{w}| \geq 0$ and $t \geq 0$

$$
\left\|w_{x x}\right\|_{L^{p}} \leq C_{p} \min \left(|\tilde{w}|, t^{-1}\right) .
$$

(iv) If $w_{-}>0$, for all $x \leq 0$ and $t \geq 0$,

$$
\begin{aligned}
\left|w(x, t)-w_{-}\right| & \leq|\tilde{w}| \exp \left\{-2\left(|x|+\left|w_{-}\right| t\right)\right\} \\
\left|w_{x}(x, t)\right| & \leq 2|\tilde{w}| \exp \left\{-2\left(|x|+\left|w_{-}\right| t\right)\right\} .
\end{aligned}
$$

(v) If $w_{+}<0$, for all $x \geq 0$ and $t \geq 0$,

$$
\begin{aligned}
\left|w(x, t)-w_{+}\right| & \leq|\tilde{w}| \exp \left\{-2\left(|x|+\left|w_{+}\right| t\right)\right\} \\
\left|w_{x}(x, t)\right| & \leq 2|\tilde{w}| \exp \left\{-2\left(|x|+\left|w_{+}\right| t\right)\right\} .
\end{aligned}
$$

(vi) $\lim _{t \rightarrow+\infty} \sup _{x \in R}\left|w(x, t)-w^{r}(x / t)\right|=0$.

Proof. Although the proof is in [11], we prove (iii) for completeness. By the method of characteristics, the solution to $(2.4)$ is expressed by

$$
w(x, t)=w_{0}\left(x_{0}(x, t)\right)
$$

where $x_{0}(x, t)$ is given by

$$
x=x_{0}(x, t)+w_{0}\left(x_{0}(x, t)\right) t .
$$

Since $w_{0}$ is strictly increasing and smooth, $x_{0}(x, t)$ exists for any nonnegative $t$ and $x \in R^{1}$ and is smooth. The relation (2.6) implies

$$
\partial x_{0}(x, t) / \partial x=\left(1+w_{0}^{\prime}\left(x_{0}\right) t\right)^{-1} \text {, }
$$

from which we have $w_{x x}=w_{0}^{\prime \prime}\left(x_{0}\right) /\left(1+w_{0}^{\prime}\left(x_{0}\right) t\right)^{3}$. Therefore,

$$
\begin{aligned}
\int_{-\infty}^{\infty}\left|w_{x x}(x, t)\right|^{p} d x & =\int_{-\infty}^{\infty}\left|w_{x x}(x, t)\right|^{p}\left(\frac{\partial x_{0}(x, t)}{\partial x}\right)^{-1} d x_{0} \\
& =\int_{-\infty}^{\infty}\left|w_{0}^{\prime \prime}\left(x_{0}\right)\right|^{p}\left(1+w_{0}^{\prime}\left(x_{0}\right) t\right)^{1-3 p} d x_{0}
\end{aligned}
$$


which yields the first inequality

$$
\left\|w_{x x}\right\|_{L^{p}}^{p} \leq\left\|w_{0}^{\prime \prime}\right\|_{L^{p}}^{p} \leq C_{p}^{p}|\tilde{w}|^{p} .
$$

For the second inequality, we divide the integral into two parts

$$
\left\|w_{x x}\right\|_{L^{p}}^{p}=\int_{x_{0} \geq 0}+\int_{x_{0} \leq 0}=I_{1}+I_{2} .
$$

Then, introducing $y=w_{0}^{\prime}\left(x_{0}\right) t$, we estimate $I_{1}$ in the following way:

$$
\begin{aligned}
I_{1} & =\int_{0}^{w_{0}^{\prime}(0) t}\left|w_{0}^{\prime \prime}\left(x_{0}\right)\right|^{p-1}(1+y)^{1-3 p} t^{-1} d y \\
& \leq 2^{p-1} t^{-p} \int_{0}^{w_{0}^{\prime}(0) t} y^{p-1}(1+y)^{1-3 p} d y \\
& \leq C_{p}^{p} t^{-p} .
\end{aligned}
$$

We estimate $I_{2}$ in a similar way.

Now we construct a smooth approximate solution $(\varphi, \psi)$ of $\left(U^{r}, V^{r}\right)$ as follows. Let $\left(U_{m}, V_{m}\right) \in R_{1}\left(U_{-}, V_{-}\right)$be the constant state satisfying $\left(U_{+}, V_{+}\right) \in$ $R_{2}\left(U_{m}, V_{m}\right)$. We denote by $w_{1}$ (resp. $\left.w_{2}\right)$ the solution of $(2.4)$ with $w_{-}=$ $\lambda_{1}\left(V_{-}\right)$and $w_{+}=\lambda_{1}\left(V_{m}\right)$ (resp. $w_{-}=\lambda_{2}\left(V_{m}\right)$ and $w_{+}=\lambda_{2}\left(V_{+}\right)$). Then, we define $\left(\varphi_{1}, \psi_{1}\right)$ (resp. $\left.\left(\varphi_{2}, \psi_{2}\right)\right)$ by

$$
\begin{array}{cl}
\lambda_{1}\left(\psi_{1}\right)=w_{1} & \left(\text { resp. } \lambda_{2}\left(\psi_{2}\right)=w_{2}\right) \\
\varphi_{1}=-\int_{V_{-}}^{\psi_{1}} \lambda_{1}(s) d s+U_{-} & \left(\text {resp. } \varphi_{2}=-\int_{V_{m}}^{\psi_{2}} \lambda_{2}(s) d s+U_{m}\right) .
\end{array}
$$

Now we set

$$
(\varphi, \psi)(x, t)=\left(\varphi_{1}+\varphi_{2}-U_{m}, \psi_{1}+\psi_{2}-V_{m}\right)(x, t) .
$$

From (2.7) we see

$$
\lambda^{\prime}\left(\psi_{i}\right) \psi_{i t}=w_{i t}, \quad \lambda^{\prime}\left(\psi_{i}\right) \psi_{i x}=w_{i x},
$$

where $i=1,2$. Then, (2.4) implies $\psi_{i t}+\lambda\left(\psi_{i}\right) \psi_{i x}=0$. Since $\varphi_{i x}=$ $-\lambda\left(\psi_{i}\right) \psi_{i x}$, we obtain $\psi_{i t}=\varphi_{i x}$. Similarly,

$$
\varphi_{i t}=-\lambda\left(\psi_{i}\right) \psi_{i t}=\lambda^{2}\left(\psi_{i}\right) \psi_{i x}=p \sigma^{\prime}\left(\psi_{i}\right) \psi_{i x} .
$$

From this derivation it is easy to see that $(\varphi, \psi)$ satisfies the system

$$
\psi_{t}=\varphi_{x}, \quad \varphi_{t}=\rho \sigma(\psi)_{x}+p(\psi)_{x},
$$

where

$$
p(\psi)=\rho\left(\sigma\left(\psi_{1}\right)+\sigma\left(\psi_{2}\right)-\sigma(\psi)-\sigma\left(v_{m}\right)\right) .
$$

Then, $(\varphi, \psi)$ satisfies the following

Lemma 2.2. (i) $\psi_{t} \leq 0$, for all $x \in R$ and $t \geq 0$.

(ii) There exists $C$ and $\alpha(\geq 0)$ such that for all $t \geq 0, x \in R$, and $\delta$ $\left(0 \leq \delta \leq \delta_{0}\right)$,

$$
\begin{gathered}
\left|\psi_{x}\right| \leq C\left|\psi_{t}\right|, \quad\left|\psi_{t}\right| \leq C \delta \\
\left|\psi_{x x}\right| \leq C\left|\psi_{t}\right|, \quad\left|\psi_{x t}\right| \leq C\left|\psi_{t}\right|, \quad\left|\psi_{x x t}\right| \leq C\left|\psi_{t}\right| \\
\left|p_{x}\right| \leq C \delta \exp \{-\alpha(|x|+t)\}
\end{gathered}
$$


(iii) For $p(1 \leq p<\infty)$, there exists $C_{p}$ such that for all $t \geq 0$ and $\delta$ $\left(0 \leq \delta \leq \delta_{0}\right)$,

$$
\left\|\psi_{x}\right\|_{L^{p}} \leq C_{p} \delta^{1 / p}(1+t)^{-1+1 / p} .
$$

(iv) For all $p \quad(1 \leq p<\infty)$, there exist $C_{p}$ and $C$ such that for all $t \geq 0$ and $\delta\left(0 \leq \delta \leq \delta_{0}\right)$,

$$
\begin{gathered}
\left\|\psi_{x x}\right\|_{L^{p}} \leq C_{p} \min \left(\delta,(1+t)^{-1}\right) \leq C \frac{\delta^{\alpha}}{(1+t)^{1-\alpha}}, \quad 0<\alpha<1, \\
\left\|\psi_{x t}\right\|_{L^{p}} \leq C_{p} \sum_{i=1}^{2}\left\{\left\|\psi_{i x x}\right\|_{L^{p}}+\left(\left\|\psi_{i x}\right\|_{L^{2 p}}\right)^{2}\right\} .
\end{gathered}
$$

(v) $\lim _{t \rightarrow+\infty} \sup _{x \in R}\left|(\varphi, \psi)(x, t)-\left(U^{r}, V^{r}\right)(x / t)\right|=0$.

We can show the above results using Lemma 2.1 and the computation carried out from (2.7) through (2.10).

Since we consider the nonlinear perturbation of $(\varphi, \psi)$, it is convenient to change the dependent variables. We introduce the perturbation $(u, v)$ from $(\varphi, \psi)$ as

$$
(U, V)(x, t)=(u+\varphi, v+\psi)(x, t), \quad\left(u_{0}, v_{0}\right)( \pm \infty)=0,
$$

where $\left(u_{0}, v_{0}\right)(x)=(u, v)(x, 0)$. Substituting the above $(U, V)$ in (1.9) and subtracting (2.10) from it, we obtain

$$
\left\{\begin{array}{l}
v_{t}=u_{x} \\
u_{t}=\{\chi(v+\psi)-\chi(\psi)\}_{x}+\int_{0}^{t} a(t-\tau) \phi(v+\psi)_{x t} d \tau+\Phi,
\end{array}\right.
$$

with the initial data

$$
u(x, 0)=u_{0}(x), \quad v(x, 0)=v_{0}(x),
$$

where

$$
\Phi=-p_{x}+a(t) \phi\left(v_{0}+\psi_{0}\right)_{x}+h .
$$

Then, adding and subtracting $\int_{0}^{t} a(t-\tau) \phi(\psi)_{x t} d \tau$, we have

$$
\left\{\begin{aligned}
v_{t}= & u_{x}, \\
u_{t}= & (\chi(v+\psi)-\chi(\psi))_{x}+\int_{0}^{t} a(t-\tau)(\phi(v+\psi)-\phi(\psi))_{x t} d \tau \\
& +\int_{0}^{t} a(t-\tau) \phi(\psi)_{x t} d \tau+\Phi .
\end{aligned}\right.
$$

Integrating the first memory term on the right-hand side, we obtain

$$
\left\{\begin{array}{l}
v_{t}=u_{x} \\
u_{t}=(\sigma(v+\psi)-\sigma(\psi))_{x}+\int_{0}^{t} a^{\prime}(t-\tau)(\phi(v+\psi)-\phi(\psi))_{x} d \tau+\Psi
\end{array}\right.
$$

where

$$
\Psi=\int_{0}^{t} a(t-\tau) \phi(\psi)_{x t} d \tau+a(t) \phi\left(\psi_{0}\right)_{x}+h-p_{x}
$$




\section{KeRNELS}

In this section, we summarize the properties of kernels which will appear in this paper. We omit most of the proofs of lemmas appearing in this section, since they are available in [2 and 4].

We now discuss the relevant aspects of the Volterra equation. Let $b \in$ $L_{\text {loc }}^{1}[0, \infty)$ be given and consider the linear Volterra equation

$$
y(t)+\int_{0}^{t} b(t-\tau) y(\tau) d \tau=g(t), \quad t \geq 0 .
$$

For each $g \in L_{\mathrm{loc}}^{1}[0, \infty)$, (3.1) has a unique solution $y \in L_{\mathrm{loc}}^{1}[0, \infty)$. This solution is given by

$$
y(t)=g(t)+\int_{0}^{t} p(t-\tau) g(\tau) d \tau, \quad t \geq 0,
$$

where $p$ is the unique solution of the resolvent equation

$$
p(t)+\int_{0}^{t} b(t-\tau) p(\tau) d \tau=-b(t), \quad t \geq 0 .
$$

As we will see, it is important to know the conditions under which a given kernel is in $L^{1}(0, \infty)$.

First, we define the resolvent kernel $k$ associated with $a^{\prime}$ as the unique solution to

$$
\sigma^{\prime}\left(v_{-}\right) k(t)+\int_{0}^{t} a^{\prime}(t-\tau) \phi^{\prime}\left(v_{-}\right) k(\tau) d \tau=-\phi\left(v_{-}\right) a^{\prime}(t), \quad t \geq 0 .
$$

The kernel $k$ will be used to express $v_{x}$ and $v_{x x}$ in terms of $u_{t}$ and $v_{t t}$, respectively, in $\S 5$. The requirement for $k \in L^{1}(0, \infty)$ follows from

Lemma 3.1. If (1.5) and (1.6) hold, then the solution $k$ of (3.4) belongs to $L^{1}(0, \infty)$. See Lemma 3.2 of [2] for the proof.

Next, we discuss the resolvent kernel $r$ associated with $-a^{\prime \prime}$. This kernel satisfies

$$
r(t)-\int_{0}^{t} a^{\prime \prime}(t-\tau) r(\tau) d \tau=a^{\prime \prime}(t), \quad t \geq 0
$$

This kernel itself does not belong to $L^{1}(0, \infty)$. Nevertheless, $r$ is the sum of a constant and a kernel belonging to $L^{1}(0, \infty)$.

Lemma 3.2. Suppose that (1.5), (1.6), and (1.8) hold. Then, the solution of (3.5) can be expressed as

$$
r(t)=\frac{1}{a(0)}+R(t), \quad \forall t \geq 0,
$$

where $R \in L^{1}(0, \infty)$.

The proof is given in Lemma 3.2 of [4].

We define another kernel $M$ by

$$
M(t)=-\int_{t}^{\infty} R(s) d s, \quad \forall t \geq 0 .
$$

This kernel has the following properties. 
Lemma 3.3. Suppose that (1.5), (1.6), and (1.8) hold. Then, the kernel $M$ satisfies

$$
M \in A C[0, \infty) \cap L^{1}(0, \infty), \quad M(0)=1-a^{\wedge}(0) / a(0)^{2}<1 .
$$

The second property will play an important role in $\S 5$; see Lemma 2.4 of [4] for the proof.

Lastly, in the next lemma we state the mechanism which induces the dissipative effect of the memory term. For this we introduce some notations. First for $b \in L_{\text {loc }}^{1}[0, \infty)$, we set

$$
Q(z, t, b)=\int_{0}^{t} \int_{-\infty}^{\infty} z(x, s) \int_{0}^{s} b(s-\tau) z(x, \tau) d \tau d x d s, \quad \forall t \in[0, T]
$$

for every $T>0$ and every $z \in C\left([0, T] ; L^{2}(R)\right)$. We also define, for $T>0$ and $0<h<T$, the forward difference operator $\Delta_{h}$ of stepsize $h$ in the time variable by

$$
\Delta_{h} z(x, t)=z(x, t+h)-z(x, t), \quad \forall x \in R, t \in[0, T-h]
$$

for every $z \in C\left([0, T] ; L^{2}(R)\right)$. Then, we have

Lemma 3.4. Suppose that (1.5) holds. Then, there exists a constant $\kappa>0$ such that

$$
\begin{aligned}
& \int_{0}^{t} \int_{-\infty}^{\infty} z_{t}(x, t)^{2} d x d s \\
& \leq \kappa \int_{-\infty}^{\infty} z(x, 0)^{2} d x+\kappa Q\left(z_{t}, t, a\right)+\kappa \liminf _{h \downarrow 0} \frac{1}{h^{2}} Q\left(\Delta_{h} z_{t}, t, a\right), \\
& \forall t \in[0, T),
\end{aligned}
$$

for every $T>0$ and every $z \in C^{1}\left([0, T] ; L^{2}(R)\right)$.

Proof. We use the identity

$$
\begin{aligned}
a(0) \Delta_{h} z(x, t)= & a(t) \Delta_{h} z(x, 0)+\int_{0}^{t} a(t-\tau) \Delta_{h} z_{t}(x, \tau) d \tau \\
& -\int_{0}^{t} a^{\prime}(t-\tau) \Delta_{h} z(x, \tau) d \tau .
\end{aligned}
$$

Squaring the above identity and integrating over $x$ and $t$, we have

$$
\begin{aligned}
& a(0)^{2} \int_{0}^{t} \int_{-\infty}^{\infty}\left\{\Delta_{h} z(x, s)\right\}^{2} d x d s \\
& \leq 3\left\{\int_{0}^{\infty} a(t)^{2} d t\right\}\left\{\int_{-\infty}^{\infty} \Delta_{h} z(x, 0)^{2} d x\right\} \\
&+3 \int_{0}^{t} \int_{-\infty}^{\infty}\left\{\int_{0}^{s} a(s-\tau) \Delta_{h} z_{t}(x, \tau) d \tau\right\}^{2} d x d s \\
&+3 \int_{0}^{t} \int_{-\infty}^{\infty}\left\{\int_{0}^{s} a^{\prime}(s-\tau) \Delta_{h} z(x, \tau) d \tau\right\}^{2} d x d s .
\end{aligned}
$$

Note that (1.5) implies $a(0)>0, a \in L^{2}(0, \infty)$, and that there exists a positive constant $C$ such that

$$
0 \leq Q(v, t, e) \leq Q(v, t, a), \quad \forall t \in[0, T]
$$


for every $T>0$ and every $v \in C^{1}\left([0, T] ; L^{2}(R)\right)$, where $e(t)=e^{-t}, t \geq 0$. From (3.11), (3.12) and Lemma 4.2 of [12], we have the desired result.

\section{MAIN Result}

In this section we state the theorem which we shall prove in the next section and state the lemma establishing the existence of local solutions.

In what follows, we will use $\|\cdot\|$ to denote the $L^{2}$ norm unless it is explicitly specified. Regarding the initial data $u_{0}$ and $v_{0}$ in (2.15) and $h$, we require that

$$
\begin{gathered}
u_{0}, u_{0}^{\prime}, u_{0}^{\prime \prime}, v_{0}, v_{0}^{\prime}, v_{0}^{\prime \prime} \in L^{2}(R), \\
h, h_{x}, h_{t} \in C\left([0, \infty) ; L^{2}(R)\right) \cap L^{\infty}\left([0, \infty) ; L^{2}(R)\right), \\
\|h\|_{L^{1}}^{4 / 3} \in L^{1}[0, \infty), \quad h, h_{x}, h_{t}, h_{x t} \in L^{2}\left([0, \infty) ; L^{2}(R)\right) .
\end{gathered}
$$

To measure the size of the initial perturbation and $h$, we denote

$$
\Pi_{0}=\int_{-\infty}^{\infty}\left\{u_{0}^{2}+\left(u_{0}^{\prime}\right)^{2}+\left(u_{0}^{\prime \prime}\right)^{2}+v_{0}^{2}+\left(v_{0}^{\prime}\right)^{2}+\left(v_{0}^{\prime \prime}\right)^{2}\right\} d x
$$

and

$$
\begin{aligned}
H= & \sup _{t \geq 0} \int_{-\infty}^{\infty}\left\{h^{2}+h_{x}^{2}+h_{t}^{2}\right\}(x, t) d x \\
& +\int_{0}^{t} \int_{-\infty}^{\infty}\left\{h^{2}+h_{x}^{2}+h_{t}^{2}+h_{x t}^{2}\right\}(x, s) d x d s \\
& +\int_{0}^{\infty}\left(\int_{-\infty}^{\infty}|h(x, t)| d x\right)^{4 / 3} d t .
\end{aligned}
$$

Then, we have the following

Proposition 4.1. Suppose that $\sigma$ and $\phi$ satisfy (1.4) and (1.12), that a(t) satisfies (1.5), (1.6), and (1.7), and that $u_{0}, v_{0}$, and $h$ satisfy (4.1) and (4.2). Then, there exists a constant $\varepsilon>0$ such that if

$$
\delta+\Pi_{0}+H \leq \varepsilon,
$$

the initial value problem (2.17), (2.15) has a unique global solution $(u, v) \in$ $C^{1}(R \times[0, \infty))$ satisfying

$$
\begin{aligned}
& u, v, u_{t}, v_{t}, v_{x}, u_{t t}, v_{t t}, v_{x t}, v_{x x} \\
& \quad \in C\left([0, \infty) ; L^{2}(R)\right) \cap L^{\infty}\left([0, \infty) ; L^{2}(R)\right) .
\end{aligned}
$$

Furthermore,

$$
\begin{gathered}
u_{t}, v_{x}, v_{t}, u_{t t}, v_{t t}, v_{x t}, v_{x x} \in L^{2}\left([0, \infty) ; L^{2}(R)\right), \\
u_{t}, v_{t}, v_{x} \rightarrow 0 \text { in } L^{2}(R) \quad \text { as } t \rightarrow \infty .
\end{gathered}
$$

Proposition 4.2. Proposition 4.1 implies Theorem 1.1.

Proof. Note that

$$
\begin{aligned}
\left\|\left(u_{0}, v_{0}\right)\right\| & \leq\left\|\left(U_{0}-U_{0}^{r}, V_{0}-V_{0}^{r}\right)\right\|+\left\|\left(\varphi(x, 0)-U_{0}^{r}, \psi(x, 0)-V_{0}^{r}\right)\right\| \\
& \leq\left\|\left(U_{0}-U_{0}^{r}, V_{0}-V_{0}^{r}\right)\right\|+C \delta \\
\left\|\left(u_{0 x}, v_{0 x}\right)\right\|_{1} & \leq\left\|\left(U_{0 x}, V_{0 x}\right)\right\|_{1}+\left\|\left(\varphi(x, 0)_{x}, \psi(x, 0)_{x}\right)\right\|_{1} \\
& \leq\left\|\left(U_{0 x}, V_{0 x}\right)\right\|_{1}+C \delta .
\end{aligned}
$$


Therefore, $\Pi_{0}$ is bounded by $\Sigma_{0}$. Also note that

$$
\begin{gathered}
\sup _{x \in R}\left|(U, V)-\left(U^{r}, V^{r}\right)\right| \leq \sup _{x \in R}|(u, v)|+\sup _{x \in R}\left|(\varphi, \psi)-\left(U^{r}, V^{r}\right)\right|, \\
\sup _{x \in R} u^{2} \leq 2\|u\|\left\|u_{x}\right\|, \quad \text { and } \sup _{x \in R} v^{2} \leq 2\|v\|\left\|v_{x}\right\| .
\end{gathered}
$$

Hence, (4.6), (4.8), and (v) in Lemma 2.2 imply (1.18) in Theorem 1.1.

In order to prove Proposition 4.1 we need the local solution in time. This is provided by the following

Lemma 4.1. Suppose that the assumptions (1.4), (1.12), (1.5), (1.6), (1.7), (4.1), and (4.2) are satisfied. Then, there is a unique solution $(u, v) \in C^{1}\left(\left[0, T_{0}\right) \times R\right)$ of (2.17) defined on a maximal interval $\left[0, T_{0}\right), T_{0} \leq \infty$, such that for $T \in$ $\left[0, T_{0}\right)$

$$
\begin{aligned}
& u, v, u_{t}, v_{t}, v_{x}, u_{t t}, v_{t t}, v_{x t}, v_{x x} \\
& \quad \in C\left([0, T] ; L^{2}(R)\right) \cap L^{\infty}\left([0, T] ; L^{2}(R)\right) .
\end{aligned}
$$

Furthermore, if $T_{0}<\infty$, then

$$
\begin{array}{r}
\int_{-\infty}^{\infty}\left\{u^{2}+v^{2}+u_{t}^{2}+v_{t}^{2}+v_{x}^{2}+u_{t t}^{2}+v_{t t}^{2}+v_{x t}^{2}+v_{x x}^{2}\right\}(x, t) d x \rightarrow \infty \\
\text { as } t \rightarrow T_{0} .
\end{array}
$$

The proof is essentially the same as in [2]. Therefore, we omit the details.

\section{Proof of Proposition 4.1}

The proof is based on the energy method. In what follows, $C$ will denote generic positive constants which may be large. It is convenient to define

$$
\begin{gathered}
E(t)=\sup _{0 \leq s \leq t} \int_{-\infty}^{\infty}\left\{u^{2}+v^{2}+u_{t}^{2}+v_{t}^{2}+v_{x}^{2}+u_{t t}^{2}+v_{t t}^{2}+v_{x t}^{2}+v_{x x}^{2}\right\}(x, s) d x, \\
F(t)=\int_{0}^{t} \int_{-\infty}^{\infty}\left\{u_{t}^{2}+v_{t}^{2}+v_{x}^{2}+u_{t t}^{2}+v_{t t}^{2}+v_{x t}^{2}+v_{x x}^{2}\right\}(x, s) d x d s .
\end{gathered}
$$

Choosing a sufficiently small positive number $\mu$, we modify $\sigma$ smoothly outside the interval $\left[\min \left(v_{-}, v_{+}\right)-\mu, v_{m}+\mu\right.$ ] so that $\sigma^{\prime \prime}$ is identically zero outside the interval $\left[\min \left(v_{-}, v_{+}\right)-2 \mu, v_{m}+2 \mu\right.$ ] and choose $\underline{\sigma}$ and $\bar{\sigma}$ such that

$$
0<\underline{\sigma} \leq \sigma^{\prime}(\xi) \leq \bar{\sigma}, \quad \forall \xi \in R
$$

Since $\sigma^{\prime \prime}$ is identically zero outside the above interval, $\sigma^{\prime \prime}(\xi)$ and $\sigma^{\prime \prime \prime}(\xi)$ have finite maxima in $\xi \in R$. This modification will not alter the problem, since we will see posteriori that $v \in\left[\min \left(v_{-}, v_{+}\right)-\mu, v_{m}+\mu\right]$.

As is discussed in the previous section, we normalize $a(t)$ so that $a^{\prime}(0)=-1$. After the differentiation of $(2.17 \mathrm{~b})$ with respect to $t$, we have

$$
\left\{\begin{aligned}
v_{t}= & u_{x} \\
u_{t t}= & (\sigma(v+\psi)-\sigma(\psi))_{x t}-(\phi(v+\psi)-\phi(\psi))_{x} \\
& +\int_{0}^{t} a^{\prime \prime}(t-\tau)(\phi(v+\psi)-\phi(\psi))_{x} d \tau+\Psi_{t} .
\end{aligned}\right.
$$


Using (3.5) and Lemma 3.2 to solve (5.1) for $(\phi(v+\psi)-\phi(\psi))_{x}$, we obtain

$$
\left\{\begin{aligned}
v_{t}=u_{x}, & \\
u_{t t}+\alpha u_{t}= & (\sigma(v+\psi)-\sigma(\psi))_{x t}+\alpha(\chi(v+\psi)-\chi(\psi))_{x} \\
& +\Psi_{t}+\alpha \Psi+\left[R *\left\{(\sigma(v+\psi)-\sigma(\psi))_{x}-u_{t}+\Psi\right\}\right]_{t}
\end{aligned}\right.
$$

where $\alpha=1 / a(0)$.

First, we obtain an estimate for

$$
\int_{-\infty}^{\infty}\left\{u^{2}+v^{2}\right\}(x, t) d x+\int_{0}^{t} \int_{-\infty}^{\infty}\left\{\left|\psi_{t}\right| v^{2}+u_{t}^{2}+v_{t}^{2}+v_{x}^{2}\right\}(x, s) d x d s
$$

For this purpose, we multiply $(5.2 \mathrm{~b})$ by $u$ and integrate over $[0, t] \times R, 0<$ $t<T$. Using integrations by parts with respect to $x$ and $t$, we obtain

$$
\begin{aligned}
\alpha \int_{-\infty}^{\infty} & \left\{\frac{1}{2} u^{2}+W(v, \psi)\right\}(x, t) d x+\alpha \int_{0}^{t} \int_{-\infty}^{\infty} Z(v, \psi) \psi_{t}(x, s) d x d s \\
& +\int_{0}^{t} \int_{-\infty}^{\infty} \sigma^{\prime}(v+\psi) v_{t}^{2}(x, s) d x d s-\int_{0}^{t} \int_{-\infty}^{\infty} u_{t}^{2}(x, s) d x d s \\
= & \int_{-\infty}^{\infty}\left\{\frac{1}{2} \alpha u_{t}^{2}+\alpha W(v, \psi)+u u_{t}-\Psi u\right\}(x, 0) d x \\
& +\int_{-\infty}^{\infty}\left\{\Psi u-u u_{t}\right\}(x, t) d x \\
& +\int_{-\infty}^{\infty} u\left[R *\left\{(\sigma(v+\psi)-\sigma(\psi))_{x}-u_{t}+\Psi\right\}\right](x, t) d x \\
& -\int_{0}^{t} \int_{-\infty}^{\infty}\left(\sigma^{\prime}(v+\psi)-\sigma^{\prime}(\psi)\right) \psi_{t} v_{t}(x, s) d x d s \\
& -\int_{0}^{t} \int_{-\infty}^{\infty} u_{t}\left[R *\left\{(\sigma(v+\psi)-\sigma(\psi))_{x}-u_{t}+\Psi\right\}\right](x, s) d x d s \\
& +\int_{0}^{t} \int_{-\infty}^{\infty} \Psi\left(\alpha u-u_{t}\right)(x, s) d x d s,
\end{aligned}
$$

where

$$
\begin{gathered}
W(v, \psi)=\int_{\psi}^{v+\psi} \chi(z) d z-\chi(\psi) v, \\
Z(v, \psi)=\chi(\psi)-\chi(v+\psi)+\chi^{\prime}(\psi) v .
\end{gathered}
$$

To eliminate the term $\int_{0}^{t} \int_{-\infty}^{\infty} u_{t}^{2}(x, s) d x d s$, we make another identity. Multiplying (2.14) by $u_{t}$ and integrating over $[0, t] \times R, 0<t<T$, we have 


$$
\begin{aligned}
\int_{0}^{t} \int_{-\infty}^{\infty} & u_{t}^{2}(x, s) d x d s-\int_{0}^{t} \int_{-\infty}^{\infty} \chi^{\prime}(v+\psi) v_{t}^{2}(x, s) d x d s \\
= & -\int_{-\infty}^{\infty}(\chi(v+\psi)-\chi(\psi)) v_{t}(x, t) d x \\
& +\int_{-\infty}^{\infty}(\chi(v+\psi)-\chi(\psi)) v_{t}(x, 0) d x \\
& -\int_{0}^{t} \int_{-\infty}^{\infty}\left(\chi^{\prime}(v+\psi)-\chi^{\prime}(\psi)\right) \psi_{t} v_{t}(x, s) d x d s \\
& +\int_{0}^{t} \int_{-\infty}^{\infty} u_{t}\left[a * \phi(v+\psi)_{x t}\right](x, s) d x d s \\
& +\int_{0}^{t} \int_{-\infty}^{\infty} \Phi u_{t}(x, s) d x d s
\end{aligned}
$$

Adding (5.4) and (5.5), we obtain

$$
\begin{aligned}
\alpha \int_{-\infty}^{\infty} & \left\{\frac{1}{2} u^{2}+W(v, \psi)\right\}(x, t) d x \\
& +\int_{0}^{t} \int_{-\infty}^{\infty}\left\{\alpha Z(v, \psi) \psi_{t}+a(0) \phi^{\prime}(v+\psi) v_{t}^{2}\right\}(x, s) d x d s \\
= & \int_{-\infty}^{\infty}\left\{\frac{1}{2} \alpha u^{2}+\alpha W(v, \psi)+u u_{t}-\Psi u+(\chi(v+\psi)-\chi(\psi)) v_{t}\right\}(x, 0) d x \\
& +I_{1}+I_{2}+I_{3}+I_{4}+I_{5}+I_{6}+I_{7},
\end{aligned}
$$

where

$$
\begin{gathered}
\left.I_{1}=\int_{-\infty}^{\infty}\left\{\Psi u-u u_{t}-(\chi(v+\psi)-\chi(\psi)) v_{t}\right)\right\}(x, t) d x \\
I_{2}=\int_{-\infty}^{\infty} u\left[R *\left\{(\sigma(v+\psi)-\sigma(\psi))_{x}-u_{t}+\Psi\right\}\right](x, t) d x \\
I_{3}=-\int_{0}^{t} \int_{-\infty}^{\infty} a(0)\left(\phi^{\prime}(v+\psi)-\phi^{\prime}(\psi)\right) \psi_{t} v_{t}(x, s) d x d s \\
I_{4}=-\int_{0}^{t} \int_{-\infty}^{\infty} u_{t}\left[R *\left\{(\sigma(v+\psi)-\sigma(\psi))_{x}-u_{t}+\Psi\right\}\right](x, s) d x d s \\
I_{5}=\int_{0}^{t} \int_{-\infty}^{\infty} u_{t}\left[a * \phi(v+\psi)_{x t}\right](x, s) d x d s \\
I_{6}=\int_{0}^{t} \int_{-\infty}^{\infty}(\Phi-\Psi) u_{t}(x, s) d x d s \\
I_{7}=\int_{0}^{t} \int_{-\infty}^{\infty} \alpha \Psi u(x, s) d x d s
\end{gathered}
$$

We estimate the terms in (5.6) in the following way. First, we note that because of the assumptions on $\sigma$ and $\phi$

$$
\begin{gathered}
W(v, \psi) \geq C v^{2}, \\
Z(v, \psi) \psi_{t} \geq-C v^{2} \psi_{t} \geq 0
\end{gathered}
$$


for some positive constant $C$. The terms in $I_{1}$ through $I_{7}$ are estimated in the following way.

$$
\begin{gathered}
\left|I_{1}\right| \leq \gamma \int_{-\infty}^{\infty}\left\{u^{2}+v^{2}\right\}(x, t) d x+\frac{C}{\gamma} \int_{-\infty}^{\infty}\left\{\Psi^{2}+u_{t}^{2}+v_{t}^{2}\right\}(x, t) d x \\
\leq \gamma \int_{-\infty}^{\infty}\left\{u^{2}+v^{2}\right\}(x, t) d x+C \int_{-\infty}^{\infty}\left\{u_{t}^{2}+v_{t}^{2}\right\}(x, t) d x+C\left(\delta^{2}+H\right), \\
\left|I_{2}\right| \leq \gamma \int_{-\infty}^{\infty} u^{2}(x, t) d x+\frac{C}{\gamma} \sup _{0 \leq s \leq t, x \in R} \int_{-\infty}^{\infty}\left(u_{t}^{2}+v_{x}^{2}\right)(x, s) d x \\
+\frac{C}{\gamma} \delta^{2} \int_{-\infty}^{\infty} v^{2}(x, t) d x+\frac{C}{\gamma} \int_{-\infty}^{\infty} \Psi^{2}(x, t) d x \\
\left|I_{3}\right| \leq C \int_{0}^{t} \int_{-\infty}^{\infty}\left\{\left|\psi_{t}\right|^{3 / 2} v^{2}+\left|\psi_{t}\right| v_{t}^{2}\right\}(x, s) d x d s \\
\leq C \delta^{1 / 2} \int_{0}^{t} \int_{-\infty}^{\infty}\left\{\left|\psi_{t}\right| v^{2}+v_{t}^{2}\right\}(x, s) d x d s .
\end{gathered}
$$

In the above estimates the inequalities such as

$$
|A B| \leq \gamma A^{2}+B^{2} / 4 \gamma, \quad \forall \gamma>0
$$

have been used. There are a few terms with $\frac{1}{\gamma}$. This does not affect the estimates since we either will have these terms (see (5.16)) or can control them by choosing small values of $\delta$ or $H$. Therefore, we will include it in $C$. We estimate $I_{4}$ using the kernel $M$ defined in $\S 3$. From (2.17) we see

$$
\begin{aligned}
&(\sigma(v+\psi)-\sigma(\psi))_{x}-u_{t}+\Psi \\
&= a(0)(\phi(v+\psi)-\phi(\psi))_{x}-a(t)\left(\phi\left(v_{0}+\psi_{0}\right)-\phi\left(\psi_{0}\right)\right)_{x} \\
&-\int_{0}^{t} a(t-\tau)(\phi(v+\psi)-\phi(\psi))_{x t} d \tau .
\end{aligned}
$$

Then,

$$
\begin{aligned}
I_{4}= & a(0) \int_{0}^{t} \int_{-\infty}^{\infty} u_{t}\left[R *(\phi(v+\psi)-\phi(\psi))_{x}\right](x, s) d x d s \\
& +\int_{0}^{t} \int_{-\infty}^{\infty}[R * a](s)\left(\phi\left(v_{0}+\psi_{0}\right)-\phi\left(\psi_{0}\right)\right)_{x} u_{t}(x, s) d x d s \\
& +\int_{0}^{t} \int_{-\infty}^{\infty} u_{t}\left[R * a *(\phi(v+\psi)-\phi(\psi))_{x t}\right](x, s) d x d s
\end{aligned}
$$

Using $M$ in (3.7), we can rewrite the first term in the above relation

$$
\begin{aligned}
& R *(\phi(v+\psi)-\phi(\psi))_{x}=-M(0)(\phi(v+\psi)-\phi(\psi))_{x} \\
& \quad+M(t)\left(\phi\left(v_{0}+\psi_{0}\right)-\phi\left(\psi_{0}\right)\right)_{x}+M *(\phi(v+\psi)-\phi(\psi))_{x t}
\end{aligned}
$$


Therefore,

$$
\begin{aligned}
I_{4}= & -a(0) M(0) \int_{-\infty}^{\infty}\left(\phi^{\prime}(v+\psi)-\phi^{\prime}(\psi)\right) v_{t}(x, t) d x \\
& +a(0) M(0) \int_{-\infty}^{\infty}\left(\phi^{\prime}(v+\psi)-\phi^{\prime}(\psi)\right) v_{t}(x, 0) d x \\
& +a(0) M(0) \int_{0}^{t} \int_{-\infty}^{\infty}\left\{\phi^{\prime}(v+\psi) v_{t}^{2}+\left(\phi^{\prime}(v+\psi)-\phi^{\prime}(\psi)\right) \psi_{t} v_{t}\right\}(x, s) d x d s \\
& -\int_{0}^{t} \int_{-\infty}^{\infty}[M-R * a](s)\left(\phi\left(v_{0}+\psi_{0}\right)-\phi\left(\psi_{0}\right)\right)_{x} u_{t}(x, s) d x d s \\
& -\int_{0}^{t} \int_{-\infty}^{\infty} u_{t}\left\{[M-R * a] *(\phi(v+\psi)-\phi(\psi))_{x t}\right\}(x, s) d x d s .
\end{aligned}
$$

Denote each term in $I_{4}$ by $I_{4_{i}}, i=1, \ldots, 5$, in the order. As the terms $I_{4_{i}}$, $i=1,2,3$, can be estimated in the same way as $I_{i}, i=1,2,3$, we show an estimate for $I_{45}$.

$$
\begin{aligned}
I_{4_{s}}= & -\int_{0}^{t} \int_{-\infty}^{\infty} u_{t} \int_{0}^{s} M(s-\tau)(\phi(v+\psi)-\phi(\psi))_{x t}(x, \tau) d \tau(x, s) d x d s \\
& +\int_{0}^{t} \int_{-\infty}^{\infty} u_{t} \int_{0}^{s} R(s-\tau) \\
& \cdot \int_{0}^{\tau} a(\tau-\mu)(\phi(v+\psi)-\phi(\psi))_{x t}(x, \mu) d \mu d \tau(x, s) d x d s .
\end{aligned}
$$

Since the estimate for the first term is similar to the second term, we indicate how the second term is estimated. It should be noted that $M, R$, and $a \in$ $L^{1}(0, \infty)$ is crucial. Using Hölder's inequality and then Fubini's theorem, we have

$$
\begin{aligned}
\int_{0}^{t} \int_{-\infty}^{\infty} & \left\{u_{t} \int_{0}^{s} R(s-\tau) \int_{0}^{\tau} a(\tau-\mu)(\phi(v+\psi)-\phi(\psi))_{x t}(x, \mu) d \mu d \tau\right\} d x s \\
\leq & C\left(\int_{0}^{t} \int_{-\infty}^{\infty} u_{t}^{2}(x, s) d x d s\right)^{1 / 2} \\
\times & {\left[\int _ { 0 } ^ { t } \int _ { - \infty } ^ { \infty } \left\{\int_{0}^{s} R(s-\tau) \int_{0}^{\tau} a(\tau-\mu)\right.\right.} \\
\left.\cdot(\phi(v+\psi)-\phi(\psi))_{x t}(x, \mu) d \mu d \tau\right\} & \}^{2} d x s\right]^{1 / 2} \\
\leq & C\left(\int_{0}^{t} \int_{-\infty}^{\infty} u_{t}^{2}(x, s) d x d s\right)^{1 / 2} \\
\times & {\left[\overline { R A } \int _ { 0 } ^ { t } \left\{\int_{0}^{s}|R(s-\tau)|\right.\right.} \\
& \left.\left.\cdot\left(\int_{0}^{\tau}|a(\tau-\mu)|\left\|(\phi(v+\psi)-\phi(\psi))_{x t}\right\|^{2} d \mu\right) d \tau\right\} d s\right]^{1 / 2}
\end{aligned}
$$




$$
\begin{aligned}
& \leq C\left(\int_{0}^{t} \int_{-\infty}^{\infty} u_{t}^{2}(x, s) d x d s\right)^{1 / 2} \\
& \times(\overline{R A})\left[\int _ { 0 } ^ { t } \int _ { - \infty } ^ { \infty } \left\{v_{x t}^{2}+v_{x}^{2} v_{t}^{2}+\psi_{x}^{2} v_{t}^{2}+\psi_{t}^{2} v_{x}^{2}\right.\right. \\
& \\
& \left.\left.+\psi_{x t}^{2} v^{2}+\psi_{x}^{2} \psi_{t}^{2} v^{2}\right\}(x, s) d x d s\right]^{1 / 2},
\end{aligned}
$$

where $\bar{R}=\int_{0}^{\infty}|R(t)| d t$ and $\bar{A}=\int_{0}^{\infty}|a(t)| d t$. Therefore, we have the following estimates for $I_{4}$.

$$
\begin{aligned}
\left|I_{4}\right| \leq & C U_{0}+\varepsilon \int_{-\infty}^{\infty} v^{2}(x, t) d x+C \int_{-\infty}^{\infty} v_{t}^{2}(x, t) d x \\
& +a(0) M(0) \int_{0}^{t} \int_{-\infty}^{\infty} \phi^{\prime}(v+\psi) v_{t}^{2}(x, s) d x d s \\
& +C \delta^{1 / 2} \int_{0}^{t} \int_{-\infty}^{\infty}\left|\psi_{t}\right| v^{2}(x, s) d x d s+C \delta^{1 / 2} \int_{0}^{t} \int_{-\infty}^{\infty} v_{t}^{2}(x, s) d x d s \\
& +C \sup _{0 \leq s \leq t, x \in R} \int_{-\infty}^{\infty} u_{t}^{2}(x, t) d x \\
& +C\left(\int_{0}^{t} \int_{-\infty}^{\infty} u_{t}^{2}(x, s) d x d s\right)^{1 / 2} \\
& \times\left(\int _ { 0 } ^ { t } \int _ { - \infty } ^ { \infty } \left\{v_{x t}^{2}+v_{x}^{2} v_{t}^{2}+\psi_{x}^{2} v_{t}^{2}+\psi_{t}^{2} v_{x}^{2}\right.\right. \\
& \left.\left.+\psi_{x t}^{2} v^{2}+\psi_{x}^{2} \psi_{t}^{2} v^{2}\right\}(x, s) d x d s\right)^{1 / 2} .
\end{aligned}
$$

We should note that $M(0)<1$. Hence the fourth term in the above estimate can be absorbed in the left-hand side of (5.6). The estimate for $I_{5}$ is identical to the one for the last term in $I_{4}$. The estimate for $I_{6}$ is done as follows:

$$
\left|I_{6}\right| \leq C\left(\int_{0}^{t} \int_{-\infty}^{\infty} u_{t}^{2}(x, s) d x d s\right)^{1 / 2}\left(\int_{0}^{t} \int_{-\infty}^{\infty}\left\{\Phi^{2}+\Psi^{2}\right\}(x, s) d x d s\right)^{1 / 2} .
$$

Using Sobolev's embedding theorem, Young's inequality and Lemma 2.2 we have the following estimate for $I_{7}$ :

$$
\begin{aligned}
\left|I_{7}\right| & \leq C \int_{0}^{t}\|u\|^{1 / 2}\left\|u_{x}\right\|^{1 / 2}\|\Psi\|_{L^{1}}(s) d s \leq C E(t)^{1 / 4} \int_{0}^{t}\left(\left\|u_{x}\right\|^{2}+\|\Psi\|_{L^{1}}^{4 / 3}\right)(s) d s \\
& \leq C E(t)^{1 / 4}\left(\delta^{1 / 6}+H+F(t)\right) .
\end{aligned}
$$

Note that terms with coefficient $\varepsilon$ and $\delta$ can be absorbed in the left-hand side of (5.6) if the rarefaction waves are weak. Therefore, the above estimates, (5.6), 
(5.7), and (5.8) yield

$$
\begin{aligned}
& \int_{-\infty}^{\infty}\left\{u^{2}+v^{2}\right\}(x, t) d x+\int_{0}^{t} \int_{-\infty}^{\infty}\left|\psi_{t}\right| v^{2}(x, s) d x d s+\int_{0}^{t} \int_{-\infty}^{\infty} v_{t}^{2}(x, s) d x d s \\
& \leq C U_{0}+C \sup _{0 \leq s \leq t, x \in R} \int_{-\infty}^{\infty}\left\{u_{t}^{2}+v_{t}^{2}+v_{x}^{2}\right\}(x, s) d x \\
&+C\left(\int_{0}^{t} \int_{-\infty}^{\infty} u_{t}^{2}(x, s) d x d s\right)^{1 / 2}\left[\left(\int_{0}^{t} \int_{-\infty}^{\infty}\left\{\Phi^{2}+\Psi^{2}\right\}(x, s) d x d s\right)^{1 / 2}\right. \\
&\left.+\left(\int_{0}^{t} \int_{-\infty}^{\infty}\left\{v_{x t}^{2}+\psi_{x}^{2} v_{t}^{2}+\psi_{t}^{2} v_{x}^{2}+\psi_{x t}^{2} v^{2}+\psi_{x}^{2} \psi_{t}^{2} v^{2}\right\}(x, s) d x d s\right)^{1 / 2}\right] \\
&+C E(t)^{1 / 4}\left(\delta^{1 / 6}+H+F(t)\right) .
\end{aligned}
$$

We need estimates for $\int_{0}^{t} \int_{-\infty}^{\infty} u_{t}^{2}(x, s) d x d s$ and $\int_{0}^{t} \int_{-\infty}^{\infty} v_{x}^{2}(x, s) d x d s$. The estimate for $\int_{0}^{t} \int_{-\infty}^{\infty} u_{t}^{2}(x, s) d x d s$ is done from (5.5):

(5.11)

$$
\begin{aligned}
& \int_{0}^{t} \int_{-\infty}^{\infty} u_{t}^{2}(x, s) d x d s \\
& \leq C U_{0}+C\left(\int_{-\infty}^{\infty} v^{2}(x, t) d x\right)^{1 / 2}\left(\int_{-\infty}^{\infty} v_{t}^{2}(x, t) d x\right)^{1 / 2} \\
&+ C \int_{0}^{t} \int_{-\infty}^{\infty} v_{t}^{2}(x, s) d x d s+C \delta \int_{0}^{t} \int_{-\infty}^{\infty}\left|\psi_{t}\right| v^{2}(x, s) d x d s \\
&+ C\left(\int_{0}^{t} \int_{-\infty}^{\infty} u_{t}^{2}(x, s) d x d s\right)^{1 / 2} \\
& \cdot {\left[\left(\int_{0}^{t} \int_{-\infty}^{\infty} \Phi^{2}(x, s) d x d s\right)^{1 / 2}\right.} \\
&\left.+\left(\int_{0}^{t} \int_{-\infty}^{\infty}\left\{v_{x}^{2} v_{t}^{2}+\psi_{x}^{2} v_{t}^{2}+\psi_{t}^{2} v_{x}^{2}+\psi_{x}^{2} \psi_{t}^{2}+v_{x t}^{2}+\psi_{x t}^{2}\right\}(x, s) d x d s\right)^{1 / 2}\right] .
\end{aligned}
$$

To obtain an estimate for $\int_{0}^{t} \int_{-\infty}^{\infty} v_{x}^{2}(x, s) d x d s$, we rewrite $(2.17 \mathrm{~b})$ :

(5.12)

$$
\begin{aligned}
u_{t}= & \sigma^{\prime}\left(V_{-}\right) v_{x}+\int_{0}^{t} a^{\prime}(t-\tau) \phi^{\prime}\left(V_{-}\right) v_{x} d \tau \\
& +\left(\sigma^{\prime}(v+\psi)-\sigma^{\prime}\left(V_{-}\right)\right) v_{x}+\left(\phi^{\prime}(v+\psi)-\phi^{\prime}(\psi)\right) \psi_{x} \\
& +\int_{0}^{t} a^{\prime}(t-\tau)\left[\left(\phi^{\prime}(v+\psi)-\phi^{\prime}\left(V_{-}\right)\right) v_{x}+\left(\phi^{\prime}(v+\psi)-\phi^{\prime}(\psi)\right) \psi_{x}\right] d \tau+\Psi
\end{aligned}
$$

\section{Setting}

$$
\begin{aligned}
& G(x, t)=u_{t}-\left(\sigma^{\prime}(v+\psi)-\sigma^{\prime}\left(V_{-}\right)\right) v_{x} \\
& -\int_{0}^{t} a^{\prime}(t-\tau)\left[\left(\phi^{\prime}(v+\psi)-\phi^{\prime}\left(V_{-}\right)\right) v_{x}-\left(\phi^{\prime}(v+\psi)-\phi^{\prime}(\psi)\right) \psi_{x}\right] d \tau-\Psi
\end{aligned}
$$


and using the kernel $k$ defined in (3.4), we see that (5.12) is rewritten as

$$
\sigma^{\prime}\left(V_{-}\right) v_{x}=G+\int_{0}^{t} k(t-\tau) G(x, \tau) d \tau
$$

Squaring (5.13), integrating over $[0, t] \times R, 0<t<T$, and using Hölder's inequality and Fubini's theorem in the same way as for the estimate of $I_{4_{5}}$, we arrive at

$$
\begin{aligned}
\int_{0}^{t} \int_{-\infty}^{\infty} & v_{x}^{2}(x, s) d x d s \leq C \int_{0}^{t} \int_{-\infty}^{\infty} u_{t}^{2}(x, s) d x d s \\
& +C \int_{0}^{t} \int_{-\infty}^{\infty}\left(v^{2}+\delta^{2}\right) v_{x}^{2}(x, s) d x d s \\
& +C \int_{0}^{t} \int_{-\infty}^{\infty} \psi_{t}^{2} v^{2}(x, s) d x d s+C \int_{0}^{t} \int_{-\infty}^{\infty} \Psi^{2}(x, s) d x d s \\
\leq & C \int_{0}^{t} \int_{-\infty}^{\infty} u_{t}^{2}(x, s) d x d s+C\left(E(t)+\delta^{2}\right) \int_{0}^{t} \int_{-\infty}^{\infty} v_{x}^{2}(x, s) d x d s \\
& +\delta \int_{0}^{t} \int_{-\infty}^{\infty}\left|\psi_{t}\right| v^{2}(x, s) d x d s+\delta^{2}+H
\end{aligned}
$$

We add (5.10), (5.11), and (5.14) and use the inequality (5.9). Knowing that $\delta,\left|\psi_{t}\right|$, and $\left|\psi_{x}\right|$ are small, we have

$$
\begin{aligned}
& \int_{-\infty}^{\infty}\left\{u^{2}+v^{2}\right\}(x, t) d x+\int_{0}^{t} \int_{-\infty}^{\infty}\left|\psi_{t}\right| v^{2}(x, s) d x d s \\
& \quad+\int_{0}^{t} \int_{-\infty}^{\infty}\left\{u_{t}^{2}+v_{t}^{2}+v_{x}^{2}\right\}(x, s) d x d s \\
& \leq C U_{0}+C \sup _{0 \leq s \leq t, x \in R} \int_{-\infty}^{\infty}\left\{u_{t}^{2}+v_{t}^{2}+v_{x}^{2}\right\}(x, s) d x \\
&+C\left(\delta^{1 / 6}+H\right) E(t)^{1 / 4}+C \int_{0}^{t} \int_{-\infty}^{\infty} v_{x t}^{2}(x, s) d x d s \\
&+C\left(E(t)^{1 / 4}+E(t)+\delta^{2}\right) F(t)+C\left(\delta+\delta^{2}+H\right) .
\end{aligned}
$$

Next, we obtain an estimate for

$$
\begin{gathered}
\int_{-\infty}^{\infty}\left\{u_{t}^{2}+v_{t}^{2}+v_{x}^{2}+u_{t t}^{2}+v_{t t}^{2}+v_{x t}^{2}+v_{x x}^{2}\right\}(x, t) d x \\
+\int_{0}^{t} \int_{-\infty}^{\infty}\left\{u_{t t}^{2}+v_{t t}^{2}+v_{x t}^{2}+v_{x x}^{2}\right\}(x, s) d x d s
\end{gathered}
$$

For this purpose, first we multiply $(2.14)$ by $\phi(v+\psi)_{x t}$ and integrate over 
$[0, t] \times R, 0<t<T$. Then, we have

$$
\begin{aligned}
& \int_{-\infty}^{\infty}\left\{\frac{1}{2} \phi^{\prime}(v+\psi) v_{t}^{2}+\frac{1}{2} \chi^{\prime}(v+\psi) \phi^{\prime}(v+\psi) v_{x}^{2}\right\}(x, t) d x \\
& +Q\left(\phi(v+\psi)_{x t}, t, a\right) \\
& =\int_{-\infty}^{\infty}\left\{\frac{1}{2} \phi^{\prime}(v+\psi) v_{t}^{2}+\frac{1}{2} \chi^{\prime}(v+\psi) \phi^{\prime}(v+\psi) v_{x}^{2}\right. \\
& \left.+\phi^{\prime}(v+\psi) \psi_{t} v_{t}\right\}(x, 0) d x \\
& -\int_{-\infty}^{\infty} \phi^{\prime}(v+\psi) \psi_{t} v_{t}(x, t) d x \\
& +\int_{0}^{t} \int_{-\infty}^{\infty}\left\{\phi^{\prime \prime}(v+\psi)\left(v_{t}+\psi_{t}\right) \psi_{t} v_{t}+\phi^{\prime}(v+\psi) \psi_{t t} v_{t}\right. \\
& +\frac{1}{2} \phi^{\prime \prime}(v+\psi)\left(v_{t}+\psi_{t}\right) v_{t}^{2} \\
& +\left[\chi^{\prime \prime}(v+\psi) \phi^{\prime}(v+\psi)+\chi^{\prime}(v+\psi) \phi^{\prime \prime}(v+\psi)\right]\left(v_{t}+\psi_{t}\right) v_{x}^{2} \\
& -\chi(v+\psi) \phi^{\prime}(v+\psi) \psi_{x t} v_{x}-\left(\chi^{\prime}(v+\psi)-\chi^{\prime}(\psi)\right) \\
& \left.\cdot \phi^{\prime \prime}(v+\psi) \psi_{x}\left(v_{t}+\psi_{t}\right)\left(v_{x}+\psi_{x}\right)\right\}(x, s) d x d s \\
& +\int_{0}^{t} \int_{-\infty}^{\infty} \Phi \phi(v+\psi)_{x t}(x, s) d x d s .
\end{aligned}
$$

The terms on the right-hand side of (5.17) are rather routinely estimated. For example,

$$
\begin{aligned}
\left|\int_{-\infty}^{\infty} \phi^{\prime}(v+\psi) \psi_{t} v_{t}(x, t) d x\right| & \leq \gamma \int_{-\infty}^{\infty} v_{t}^{2}(x, t) d x+\frac{C}{\gamma} \int_{-\infty}^{\infty} \psi_{t}^{2}(x, t) d x \\
& \leq \gamma \int_{-\infty}^{\infty} v_{t}^{2}(x, t) d x+\frac{C}{\gamma} \delta
\end{aligned}
$$




$$
\begin{aligned}
& \left|\int_{0}^{t} \int_{-\infty}^{\infty} \frac{1}{2} \phi^{\prime \prime}(v+\psi)\left(v_{t}+\psi_{t}\right) v_{t}^{2}(x, s) d x d s\right| \\
& \leq C\left(\sup _{0 \leq t, x \in R}\left|v_{t}\right|+\delta\right) \int_{0}^{t} \int_{-\infty}^{\infty} v_{t}^{2}(x, s) d x d s \\
& \leq C\left(E(t)^{1 / 2}+\delta\right) \int_{0}^{t} \int_{-\infty}^{\infty} v_{t}^{2}(x, s) d x d s \\
& \left|\int_{0}^{t} \int_{-\infty}^{\infty}\left(\chi^{\prime}(v+\psi)-\chi^{\prime}(\psi)\right) \phi^{\prime \prime}(v+\psi) \psi_{x}\left(v_{t}+\psi_{t}\right)\left(v_{x}+\psi_{x}\right)(x, s) d x d s\right| \\
& \leq C \int_{0}^{t} \int_{-\infty}^{\infty}\left\{\left(\psi_{x}^{2}+\psi_{t}^{2}\right) v^{2}+\psi_{t}^{4}+\psi_{x}^{2} v_{t}^{2}+\psi_{x}^{2} v_{x}^{2}\right\}(x, s) d x d s \\
& +C \sup _{0 \leq t, x \in R}|v| \int_{0}^{t} \int_{-\infty}^{\infty}\left(v_{t}^{2}+v_{x}^{2}\right)(x, s) d x d s \\
& \leq C\left(E(t)^{1 / 2}+\delta^{2}\right) \int_{0}^{t} \int_{-\infty}^{\infty}\left(v_{t}^{2}+v_{x}^{2}\right)(x, s) d x d s \\
& +C \delta \int_{0}^{t} \int_{-\infty}^{\infty}\left|\psi_{t}\right| v^{2}(x, s) d x d s+C \delta \\
& \left|\int_{0}^{t} \int_{-\infty}^{\infty} \Phi \phi(v+\psi)_{x t}(x, s) d x d s\right| \\
& \leq C\left(\int_{0}^{t} \int_{-\infty}^{\infty} \Phi^{2}(x, s) d x d s\right)^{1 / 2} \\
& \times\left(\int_{0}^{t} \int_{-\infty}^{\infty}\left\{v_{x t}^{2}+v_{x}^{2} v_{t}^{2}+\psi_{x}^{2} v_{t}^{2}+\psi_{t}^{2} v_{x}^{2}+\psi_{x t}^{2}+\psi_{x}^{2} \psi_{t}^{2}\right\}(x, s) d x d s\right)^{1 / 2} \\
& \leq C \sqrt{H+\delta} \sqrt{F(t)}+C\left(E(t)+\delta^{2}\right) F(t)+C(H+\delta) .
\end{aligned}
$$

Combining the above estimates, we have

$$
\begin{aligned}
& \int_{-\infty}^{\infty}\left\{v_{t}^{2}+v_{x}^{2}\right\}(x, t) d x+Q\left(\phi(v+\psi)_{x t}, t, a\right) \\
& \leq C \int_{-\infty}^{\infty}\left\{v_{t}^{2}+v_{x}^{2}\right\}(x, 0) d x \\
&+C \delta \int_{0}^{t} \int_{-\infty}^{\infty}\left|\psi_{t}\right| v^{2}(x, s) d x d s+C \sqrt{H+\delta} \sqrt{F} \\
&+C\left(E(t)^{1 / 2}+E(t)+E(t)^{2}+\delta^{1 / 2}+\delta+\delta^{2}\right) F(t)+C(H+\delta) .
\end{aligned}
$$

To obtain the next identity, we apply the forward differentiating operator $\Delta_{h}$ to (2.14) and multiply it by $\Delta_{h} \phi(v+\psi)_{x t}$. Then, we integrate the resulting equation over $[0, t] \times R, 0<t<T$. After the integrations by parts, we derive it by $h^{2}$ and let $h \downarrow 0$. From this we have 


$$
\begin{aligned}
& \int_{-\infty}^{\infty} \frac{1}{2}\left\{\phi^{\prime}(v+\psi) v_{t t}^{2}+\chi^{\prime}(v+\psi) \phi^{\prime}(v+\psi) v_{x t}^{2}\right\}(x, t) d x \\
& +\lim _{h \downarrow 0} \frac{1}{h^{2}} Q\left(\Delta_{h} \phi(v+\psi)_{x t}, t, a\right) \\
& =\int_{-\infty}^{\infty} \frac{1}{2}\left\{\phi^{\prime}(v+\psi) v_{t t}^{2}+\chi(v+\psi) \phi^{\prime}(v+\psi) v_{x t}^{2}\right\}(x, 0) d x \\
& +\int_{-\infty}^{\infty} \phi(v+\psi)_{x t} a(t)\left(\phi^{\prime}\left(v_{0}+\psi_{0}\right)\left(u_{0 x}+\psi_{0 t}\right)\right)_{x}(x, t) d x \\
& -\int_{0}^{t} \int_{-\infty}^{\infty} \phi(v+\psi)_{x t} a^{\prime}(s)\left(\phi^{\prime}\left(v_{0}+\psi_{0}\right)\left(u_{0 x}+\psi_{0 x}\right)\right)_{x}(x, s) d x d s \\
& +\int_{0}^{t} \int_{-\infty}^{\infty}\left\{\frac{1}{2} \phi(v+\psi)\left(v_{t}+\psi\right) v_{t t}^{2}\right. \\
& +\frac{1}{2}\left(\chi^{\prime}(v+\psi) \phi(v+\psi)\right)_{t} v_{x t}^{2}+\chi(v+\psi) v_{x t} \psi_{x t t} \\
& -\left[\left(\chi^{\prime}(v+\psi)-\chi^{\prime}(\psi)\right) \psi_{x t}+\chi^{\prime \prime}(v+\psi)\left(v_{x} v_{t}+\psi_{t} v_{x}+\psi_{x} v_{t}\right)\right. \\
& \left.+\left(\chi^{\prime \prime}(v+\psi)-\chi^{\prime \prime}(\psi)\right) \psi_{x} \psi_{t}\right] \psi_{x t t} \\
& +\chi^{\prime}(v+\psi)\left[2 \phi^{\prime \prime}\left(v_{t}+\psi_{t}\right)\left(v_{x t}+\psi_{x t}\right)\right. \\
& \left.+\phi^{\prime \prime}(v+\psi)\left(v_{x}+\psi_{x}\right)\left(v_{t t}+\psi_{t t}\right)+\phi^{\prime \prime \prime}(v+\psi)\left(v_{t}+\psi_{t}\right)^{2}\left(v_{x}+\psi_{x}\right)\right] v_{x t} \\
& +\left[\left(\chi^{\prime}(v+\psi)-\chi^{\prime}(\psi)\right) \psi_{x t}+\chi^{\prime \prime}(v+\psi)\left(v_{x} v_{t}+\psi_{t} v_{x}+\psi_{x} v_{t}\right)\right. \\
& \left.+\left(\chi^{\prime \prime}(v+\psi)-\chi^{\prime \prime}(\psi)\right) \psi_{x} \psi_{t}\right] \\
& \cdot\left[2 \phi^{\prime \prime}(v+\psi)\left(v_{t}+\psi_{t}\right)\left(v_{x t}+\psi_{x t}\right)+\phi^{\prime \prime}(v+\psi)\left(v_{x}+\psi_{x}\right)\left(v_{t t}+\psi_{t t}\right)\right. \\
& \left.\left.+\phi^{\prime \prime \prime}(v+\psi)\left(v_{x}+\psi_{x}\right)\left(v_{t}+\psi_{t}\right)^{2}\right]\right\}(x, s) d x d s \\
& -\int_{0}^{t} \int_{-\infty}^{\infty} \boldsymbol{\Phi}_{x t} \phi(v+\psi)_{t t}(x, s) d x d s
\end{aligned}
$$

The terms on the right-hand side in (5.19) are also estimated routinely. For example,

$$
\begin{aligned}
& \left|\int_{0}^{t} \int_{-\infty}^{\infty} \Phi_{x t} \phi(v+\psi)_{t t}(x, s) d x d s\right| \\
& \quad \int_{0}^{t} \int_{-\infty}^{\infty} \Phi_{x t}\left\{\phi^{\prime \prime}(v+\psi)\left(v_{t}+\psi_{t}\right)^{2}+\phi^{\prime}(v+\psi)\left(v_{t t}+\psi_{t t}\right)\right\}(x, s) d x d s \\
& \quad \leq C\left(\int_{0}^{t} \int_{-\infty}^{\infty} \Phi_{x t}^{2}(x, s) d x d s\right)^{1 / 2}\left(\int_{0}^{t} \int_{-\infty}^{\infty} v_{t t}^{2}(x, s) d x d s\right)^{1 / 2} \\
& \quad+C \int_{0}^{t} \int_{-\infty}^{\infty}\left(\Phi_{x t}^{2}+v_{t}^{4}+\psi_{t}^{4}+\psi_{t t}^{2}\right)(x, s) d x d s \\
& \leq C \sqrt{H+\delta} \sqrt{F(t)}+C H+C E(t) F(t)+C \delta .
\end{aligned}
$$


The above estimate and other estimates imply

$$
\begin{aligned}
\int_{-\infty}^{\infty}\left\{v_{t t}^{2}\right. & \left.+v_{x t}^{2}\right\}(x, t) d x+\lim _{h \downarrow 0} \frac{1}{h^{2}} Q\left(\Delta_{h} \phi(v+\psi)_{x t}, t, a\right) \\
\leq & C \int_{-\infty}^{\infty}\left\{v_{t t}^{2}+v_{x t}^{2}\right\}(x, 0) d x \\
& +C \delta \int_{0}^{t} \int_{-\infty}^{\infty}\left|\psi_{t}\right| v^{2}(x, s) d x d s+C \sqrt{H+\delta} \sqrt{F} \\
& +C\left(E(t)^{1 / 2}+E(t)+E(t)^{3 / 2}+\delta+\delta^{2}\right) F(t)+C \delta
\end{aligned}
$$

Then, adding (5.18) and (5.21) and using (3.10), we find

$$
\begin{aligned}
& \int_{-\infty}^{\infty}\left\{v_{t}^{2}+v_{x}^{2}+v_{t t}^{2}+v_{x t}^{2}\right\}(x, t) d x+\int_{0}^{t} \int_{-\infty}^{\infty} v_{x t}^{2}(x, s) d x d s \\
& \leq C \int_{-\infty}^{\infty}\left\{v_{t}^{2}+v_{x}^{2}+v_{t t}^{2}+v_{x t}^{2}\right\}(x, 0) d x \\
&+C \delta \int_{0}^{t} \int_{-\infty}^{\infty}\left|\psi_{t}\right| v^{2}(x, s) d x d s+C \sqrt{H+\delta} \sqrt{F} \\
&+C\left(E(t)^{1 / 2}+E(t)+E(t)^{3 / 2}+E(t)^{2}+\delta^{1 / 2}+\delta+\delta^{2}\right) F(t)+C(\delta+H) .
\end{aligned}
$$

We need the estimates for $\int_{-\infty}^{\infty} u_{t}^{2}(x, t) d x$. Squaring (2.14) and integrating over $R$

(5.23)

$$
\begin{aligned}
\int_{-\infty}^{\infty} u_{t}^{2}(x, t) d x \leq C \int_{-\infty}^{\infty}\left((\chi(v+\psi)-\chi(\psi))_{x}\right)^{2}(x, t) d x \\
\quad+C \int_{-\infty}^{\infty}\left(\int_{0}^{t} a(t-\tau) \phi(v+\psi)_{x t} d \tau\right)^{2}(x, t) d x+C \int_{-\infty}^{\infty} \Phi^{2}(x, t) d x \\
\leq C H+C \int_{-\infty}^{\infty} v_{x}^{2}(x, t) d x+C \delta^{2} \int_{-\infty}^{\infty} v^{2}(x, t) d x \\
\quad+C \int_{0}^{t} \int_{-\infty}^{\infty}\left\{v_{x t}^{2}+v_{x}^{2} v_{t}^{2}+\psi_{x}^{2} v_{t}^{2}+\psi_{t}^{2} v_{x}^{2}+\psi_{x t}^{2}+\psi_{x}^{2} \psi_{t}^{2}\right\}(x, s) d x d s \\
\leq C H+C \int_{-\infty}^{\infty} v_{x}^{2}(x, t) d x+C \delta^{2} E(t) \\
\quad+C \int_{0}^{t} \int_{-\infty}^{\infty} v_{x t}^{2}(x, s) d x d s+C\left(E(t)+\delta^{2}\right) F(t)+C\left(\delta+\delta^{2}+H\right) .
\end{aligned}
$$

To obtain estimates for $u_{t t}$, we differentiate (2.14):

$$
\begin{aligned}
u_{t t}= & (\chi(v+\psi)-\chi(\psi))_{x t}+a(0) \phi(v+\psi)_{x t} \\
& +\int_{0}^{t} a^{\prime}(t-\tau) \phi(v+\psi)_{x t} d \tau+\Phi_{t} .
\end{aligned}
$$

Taking the square and integrating over $R$ and $[0, t] \times R, 0<t<T$. We 
obtain

(5.25)

$$
\begin{aligned}
& \int_{-\infty}^{\infty} u_{t t}^{2}(x, t) d x \leq C \int_{-\infty}^{\infty} v_{x t}^{2}(x, t) d x+C\left(E(t)+\delta^{2}\right) E(t) \\
& +C \int_{0}^{t} \int_{-\infty}^{\infty} v_{x t}^{2}(x, s) d x d s+C\left(E(t)+\delta^{2}\right) F(t)+C\left(\delta+\delta^{2}+H\right)
\end{aligned}
$$

$$
\begin{aligned}
& \int_{0}^{t} \int_{-\infty}^{\infty} u_{t t}^{2}(x, s) d x d s \leq C \int_{0}^{t} \int_{-\infty}^{\infty} v_{x t}^{2}(x, s) d x d s \\
& \quad+C \delta \int_{0}^{t} \int_{-\infty}^{\infty}\left|\psi_{t}\right| v^{2}(x, s) d x d s+C\left(E(t)+\delta^{2}\right) F(t)+C\left(\delta+\delta^{2}+H\right)
\end{aligned}
$$

To estimate $\int_{0}^{t} \int_{-\infty}^{\infty} v_{t t}^{2}(x, s) d x d s$, we use the identity obtained by the integration by parts and the density argument:

$$
\begin{aligned}
\int_{0}^{t} \int_{-\infty}^{\infty} v_{t t}^{2}(x, s) d x d s=\int_{0}^{t} \int_{-\infty}^{\infty} u_{x t}^{2}(x, s) d x d s \\
\quad=\int_{0}^{t} \int_{-\infty}^{\infty} u_{t t} u_{x x}(x, s) d x d s-\int_{-\infty}^{\infty} u_{t} u_{x x}(x, t) d x \\
\quad+\int_{-\infty}^{\infty} u_{t} u_{x x}(x, 0) d x \\
=\int_{0}^{t} \int_{-\infty}^{\infty} u_{t t} v_{x t}(x, s) d x d s-\int_{-\infty}^{\infty} u_{t} v_{x t}(x, t) d x \\
\quad+\int_{-\infty}^{\infty} u_{t} v_{x t}(x, 0) d x
\end{aligned}
$$

Then, from the above identity we have

$$
\begin{gathered}
\int_{0}^{t} \int_{-\infty}^{\infty} v_{t t}^{2}(x, s) d x d s \leq C U_{0}+C \int_{-\infty}^{\infty}\left\{u_{t}^{2}+v_{x t}^{2}\right\}(x, t) d x \\
+C \int_{0}^{t} \int_{-\infty}^{\infty}\left\{u_{t t}^{2}+v_{x t}^{2}\right\}(x, s) d x d s
\end{gathered}
$$

To obtain an estimate for $v_{x x}$, we differentiate (5.13):

$$
\sigma^{\prime}\left(v_{-}\right) v_{x x}=G_{x}+\int_{0}^{t} k(t-\tau) G(x, \tau)_{x} d \tau
$$

As $k \in L^{1}(0, \infty)$, we see

$$
\begin{gathered}
\int_{-\infty}^{\infty} v_{x x}^{2}(x, t) d x \\
\leq C \int_{-\infty}^{\infty} v_{t t}^{2}(x, t) d x+C\left(E(t)+\delta^{2}\right) E(t)+C\left(\delta^{2}+H\right) \\
\int_{0}^{t} \int_{-\infty}^{\infty} v_{x x}^{2}(x, s) d x d s \leq C \int_{0}^{t} \int_{-\infty}^{\infty} v_{t t}^{2}(x, s) d x d s \\
+C\left(E(t)+\delta^{2}\right) F(t)+C \delta \int_{0}^{t} \int_{-\infty}^{\infty}\left|\psi_{t}\right| v^{2}(x, s) d x d s+C H
\end{gathered}
$$


Combining the above estimates, we have

$$
\begin{aligned}
\int_{-\infty}^{\infty}\left\{u_{t}^{2}\right. & \left.+v_{t}^{2}+v_{x}^{2}+u_{t t}^{2}+v_{t t}^{2}+v_{x t}^{2}+v_{x x}^{2}\right\}(x, t) d x \\
& +\int_{0}^{t} \int_{-\infty}^{\infty}\left\{u_{t t}^{2}+v_{t t}^{2}+v_{x t}^{2}+v_{x x}^{2}\right\}(x, s) d x d s \\
\leq & C U_{0}+C\left(E(t)+\delta^{2}\right) E(t) \\
& +C \delta \int_{0}^{t} \int_{-\infty}^{\infty}\left|\psi_{t}\right| v^{2}(x, s) d x d s+C \sqrt{H+\delta} \sqrt{F} \\
& +C\left(E(t)^{1 / 2}+E(t)+E(t)^{3 / 2}+E(t)^{2}+\delta^{1 / 2}+\delta+\delta^{2}\right) F(t)+C(\delta+H) .
\end{aligned}
$$

Finally, (5.15) and (5.32) imply

$$
\begin{aligned}
E(t)+ & F(t)+\int_{0}^{t} \int_{-\infty}^{\infty}\left|\psi_{t}\right| v^{2}(x, s) d x d s \\
\leq & C U_{0}+C\left(\delta^{1 / 6}+H\right) E(t)^{1 / 4}+C\left(E(t)+\delta^{2}\right) E(t) \\
& +C \delta \int_{0}^{t} \int_{-\infty}^{\infty}\left|\psi_{t}\right| v^{2}(x, s) d x d s+C \sqrt{H+\delta} \sqrt{F} \\
& +C\left(E(t)^{1 / 4}+E(t)^{1 / 2}+E(t)+E(t)^{3 / 2}+E(t)^{2}+\delta^{1 / 2}+\delta+\delta^{2}\right) F(t) \\
& +C\left(\delta+\delta^{2}+H\right) .
\end{aligned}
$$

Therefore, as far as $E(t)<\mu$ and $\delta<\delta_{0}$, using (5.9) we finally arrive at the estimate

$$
E(t)+F(t)+\int_{0}^{t} \int_{-\infty}^{\infty}\left|\psi_{t}\right| v^{2}(x, s) d x d s \leq C\left(U_{0}+\delta_{0}+H\right) .
$$

So, if we take the initial data to be $\mu_{0}<\mu / 4 C$ and $\delta_{0}+H<\mu / 4 C$, then as far as $E(t) \leq \mu$ we have

$$
E(t)+F(t)+\int_{0}^{t} \int_{-\infty}^{\infty}\left|\psi_{t}\right| v^{2}(x, s) d x d s \leq \frac{1}{2} \mu,
$$

which shows $E(t)$ is actually smaller than $\mu$. Now invoking Lemma 4.1, we conclude that $T_{0}=\infty$. This estimate yields (4.6) and (4.7), and consequently (4.8). This completes the proof.

\section{ACKNOWLEDGMENTS}

I would like to thank Professor Tai-Ping Liu for his helpful comments. Thanks are also due to the referee for taking his time to correct my manuscript. His suggestions led to a substantial improvement in this paper. This work was supported in part by Army Grant DAAL 03-89-G-0088.

\section{REFERENCES}

1. C. M. Dafermos and J. A. Nohel, Energy methods for nonlinear hyperbolic Volterra integrodifferential equations, Comm. Partial Differential Equations 4 (1979), 219-278.

2. $\ldots$ A nonlinear hyperbolic Volterra equation in viscoelasticity, Amer. J. Math. Suppl. (1981), 87-116. 
3. J. M. Greenberg and L. Hsiao, The Riemann problem for the system $u_{t}+\sigma_{x}=0$ and $(\sigma-f(u))_{x}+(\sigma-\nu f(u))=0$, Arch. Rational Mech. Anal. 82 (1983), 87-108.

4. W. J. Hrusa and J. A. Nohel, The Cauchy problem in one-dimensional nonlinear viscoelasticity, J. Differential Equations 59 (1985), 388-412.

5. T. P. Liu, Hyperbolic conservation laws with relaxation, Comm. Math. Phys. 108 (1987), 153-175.

6. __ Nonlinear waves for viscoelasticity with fading memory, J. Differential Equations $\mathbf{7 6}$ (1988), 26-46.

7. T. P. Liu and Z. P. Xin, Nonlinear stability of rarefaction waves for compressible NavierStokes equations, Comm. Math. Phys. 118 (1988), 451-465.

8. R. C. MacCamy, An integro-differential equation with application in heat flow, Quart. Appl. Math. 35 (1977), 1-19.

9. __ A model for one-dimensional nonlinear viscoelasticity, Quart. Appl. Math. 35 (1977), 21-33.

10. _ A model Riemann problem for Volterra equations, Arch. Rational Mech. Anal. 82 (1983), 72-86.

11. A. Matsumura and K. Nishihara, Asymptotics toward the rarefaction waves of the solutions of a one-dimensional model system for compressible viscous gas, Japan J. Appl. Math. 3 (1986), 1-13.

12. O. Staffans, On a nonlinear hyperbolic Volterra equations, SIAM J. Math. Anal. 11 (1980), 793-812.

13. Z. P. Xin, Asymptotic stability of rarefaction waves for $2 \times 2$ viscous hyperbolic conservation laws, J. Differential Equations 73 (1988), 45-77.

14. Asymptotic stability of rarefaction waves for $2 \times 2$ viscous hyperbolic conservation laws-the two-mode case, J. Differential Equations 78 (1989), 191-219.

Department of Mathematics, West Virginia University, Morgantown, West Virginia 26506

E-mail address: u14930wvnvm.bitnet 